\title{
Should we genetically test everyone for haemochromatosis?
}

\author{
Katrina Allen and Robert Williamson The Murdoch Institute, Royal Children's Hospital, Melbourne, Australia
}

\begin{abstract}
The increasing availability of DNA-based diagnostic tests has raised issues about whether these should be applied to the population at large in order to identify, treat or prevent a range of diseases. DNA tests raise concerns in the community for several reasons. There is the possibility of stigmatisation and discrimination between those who test positive and those who don't. High-risk individuals may be identified for whom no proven effective intervention is possible, or conversely may test "positive" for a disease that does not eventuate. Controversy concerning prenatal diagnosis and termination of affected pregnancies may arise. Haemochromatosis, however, is a disease that is not only treatable but also preventable if those at high risk are identified presymptomatically. This paper will identify and discuss key issues regarding

DNA-based population screening for

haemochromatosis, and argue that population-based genetic screening for haemochromatosis should be supported when a number of contentious issues are addressed. In the context of a health system with limited resources haemochromatosis is the paradigm of a disorder where there is an ethical and clinical imperative to encourage presymptomatic DNA testing for all in ethnically relevant communities.
\end{abstract}

(Fournal of Medical Ethics 1999;25:209-214)

Keywords: Haemochromatosis; gene test; population screening; genetic predictive testing

\section{Introduction}

Traditionally, disease diagnosis and management are initiated by the presentation of a patient with symptoms. A number of technologies now allow medical practitioners to identify an individual's propensity to a wide range of diseases and act to prevent their onset. Examples of such preventive management range from the identification of hypothyroidism and phenylketonuria through newborn screening to prevent mental deficiency, cervical screening to identify those with earlystage cervical cancer amenable to therapy, to the cessation of smoking to prevent lung cancer. This paradigm shift from treatment to prevention has resulted from the exponential increase in the range of diagnostic tests available to the physician, including biochemical and haematological blood tests, microbiology, histology and radiology. New advances using DNA data from molecular genetics extend this by allowing medical practitioners not only to diagnose a disease at an early stage, but also to predict the likelihood of a disease developing in the future.

The increasing availability of DNA-based tests throws into relief questions of when genetic screening of the general population for common and treatable diseases is ethically sound, medically appropriate and financially cost-effective. ${ }^{1-3}$ There have been many contributions discussing the appropriate times and contexts in which to offer carrier and presymptomatic testing. ${ }^{4-6}$ Presymptomatic identification of Huntington's disease is available to high-risk families, but this disease is severe and untreatable. Carrier testing for cystic fibrosis is offered to help couples make a decision about whether to continue a pregnancy. In contrast, haemochromatosis is both preventable and treatable, and it is unlikely that couples will wish to make decisions regarding the continuation of a pregnancy based on fetal genetic status. Thus haemochromatosis is an excellent example of a genetic test that offers presymptomatic genetic identification of a preventable adult-onset disease.

Since the discovery of the gene for haemochromatosis and the ability to identify homozygotes presymptomatically, many countries are considering whether haemochromatosis is an appropriate disease for which to employ population genetic screening. Critical issues of "how", "when", and, more importantly "who" should be screened remain to be resolved.

\section{Hereditary haemochromatosis}

Hereditary haemochromatosis $(\mathrm{HH})$ is a recessively inherited metabolic disorder caused by a defect in the cells lining the intestine which leads to excessive and unregulated absorption of iron from the diet. $\mathrm{HH}$ (referred to as haemochromatosis in this paper) describes an iron overload syndrome without any external cause such as seen following repeated blood transfusions, prolonged inappropriate administration of iron, or in associ- 
ation with alcoholic liver disease. If haemochromatosis is left undiagnosed and untreated, the excess iron stored in the parenchymal cells of major organs, primarily the liver, pancreas, heart, pituitary and joints, eventually leads to severe tissue damage and premature death from liver and cardiac failure. A few grams of iron are normally stored in the liver but accumulation of more than 16 grams is associated with serious symptoms of iron overload.

Haemochromatosis has protean manifestations and a patient may present to any number of clinical specialties with signs of liver disease, heart failure, diabetes, hypogonadism, arthritis or even chronic fatigue. Because symptoms of haemochromatosis are often non-specific, have a gradual onset and can mimic other common disorders, diagnosis is frequently delayed. Liver disease may present as an abnormal elevation in liver enzymes, clinical signs of cirrhosis or hepatocellular carcinoma. ${ }^{7}$ Patients usually present with symptoms in the 5 th or 6 th decade of life though there have been reports of individuals presenting with cirrhosis as early as the 3rd decade of life. ${ }^{7}$

With the possible exception of arthritis, the clinical manifestations of $\mathrm{HH}$ can be prevented by prophylactic phlebotomy. ${ }^{7}$ Regular phlebotomy can also prevent progression of disease in patients presenting with symptomatic iron overload. ${ }^{8}$ When phlebotomy is started prior to the development of cirrhosis or diabetes, affected people have a normal life expectancy. ${ }^{9}$ Preventive phlebotomy (giving one $500 \mathrm{ml}$ unit of blood - approximately 1 gram of iron - every few months) is not usually recommended until the individual is in his/her 3rd decade, although this may change with definitive presymptomatic diagnosis. Avoidance of alcohol is also often advised for patients with $\mathrm{HH}$ since a heavy alcohol intake may be a synergistic cofactor in the development of liver fibrosis. ${ }^{10}$

The gene mutated to cause haemochromatosis, HFE, has recently been identified. ${ }^{11} \mathrm{~A}$ missense mutation (C282Y) is the most common gene defect and affects more than $85 \%$ of $\mathrm{HH}$ patients of Northern European descent, ${ }^{12-14}$ while a second mutation (H63D) is possibly associated with a less severe form of the disease. ${ }^{12}$

The prevalence of $\mathrm{HH}$ in certain populations is very high. Recent research estimates that $10 \%$ of people of Northern European ancestry are heterozygous for $\mathrm{HH}$, and one person in 300 from this ethnic group expresses the disease,${ }^{15}$ making it a significant public health problem in communities of Northern European extraction.

Environmental factors impact on whether and/or when homozygous individuals develop clinical haemochromatosis. It is well established that women with haemochromatosis usually present later in life than men, probably becaus blood loss through menstruation retards the accu? mulation of pathological iron stores. Supplemer tal iron and vitamin $C$ (which increases iron absorption) are likely to precipitate earlier phenf유 typic expression, whereas blood donation ance physiological or pathological blood loss will low the amount of iron stored in the liver and delây phenotypic expression. ${ }^{16}$ Associations betweeh several diseases (porphyria cutanea tarda, alc $\odot$ holic liver disease, non-alcoholic steatohepatitis and hepatitis B) ${ }^{17}$ and haemochromatosis have been noted. Investigations as to whether thes diseases are associated with haemochromatos expression in homozygotes are necessary.

Despite the identification of several factors that can influence the time of expression of the disease there still appears to be a subgroup of people whe are homozygous for $\mathrm{HH}$ mutations but who de not develop symptoms. ${ }^{18}$ While the clinic expression of symptomatic disease is variable, Powell et al state that approximately $90 \%$ of mats and $50-70 \%$ of female homozygotes will develo organ dysfunction and potentially life-threatening complications of haemochromatosis.

Regardless of the variability of disease manife tation in $\mathrm{HH}$, the earlier the disease is identified the better the prognosis. ${ }^{9}$ Many investigators harge shown the benefit of phlebotomy for disease prevention as well as retardation of disease progression. In contrast, the prognosis for those with cirrhosis for many years is bleak. Up to $30 \%$ of male haemochromatotics with cirrhosis wit develop hepatocellular carcinoma. ${ }^{16}$ Since regulą⿰ phlebotomy to prevent haemochromatosis is rels tively benign and the potential consequences of developing disease are so serious, it is likely thgt most homozygotes would embark on a preventive management strategy to minimise the risk of developing disease even if each has a small change of not developing symptoms.

\section{Haemochromatosis and heterozygosity} It is still not clear whether there is any phenotype associated with $\mathrm{HH}$ heterozygosity. A study of proband relatives performed before the gene was identified found that a small proportion off presumed heterozygotes (from family studies? presented with elevated serum iron measures Very few of these individuals had documente iron overload or clinical symptoms. Among tho who did, other contributing factors such alcohol abuse were present. ${ }^{19-20}$ These data suggest that clinical $\mathrm{HH}$ occurs rarely in heterozygote, and possibly only in the presence of oth environmental risk factors. 
Compound heterozygote individuals, on the other hand, can express haemochromatosis without a precipitating cofactor. Compound heterozygotes are those individuals who carry both known HH mutations, C282Y and H63D. Several large series of haemochromatosis patients have identified a small number of compound heterozygotes who express disease. These individuals probably have a lower risk of disease expression than C282Y/C282Y homozygote patients since the proportion of $\mathrm{C} 282 \mathrm{Y} / \mathrm{H} 63 \mathrm{D}$ genotypes among $\mathrm{HH}$ cases is low despite an equal frequency of carrier rate for the $\mathrm{H} 63 \mathrm{D}$ and $\mathrm{C} 282 \mathrm{Y}$ mutation. ${ }^{17}$

\section{Why screen for haemochromatosis?}

Because the presymptomatic phase of haemochromatosis is prolonged, there is a large window of opportunity in which to diagnose risk of disease well before disease onset. For those who are presymptomatic, disease prevention is possible. For those who present with early symptoms, treatment to retard disease progression is available. In contrast, untreated haemochromatosis can result in serious morbidity and early death.

Population-based screening has the potential to prevent the development of disease, but will only be effective if appropriate education and treatment strategies are implemented alongside a screening programme. Such a programme would represent a cost-effective use of the everdiminishing health care dollar. Bassett et al estimate the cost of screening in Australia to be AU $\$ 4,943-A U \$ 11,016$ per case detected depending on the screening strategy and method of confirmation of diagnosis. ${ }^{21}$ This compares favourably with other public health screening initiatives already in use such as cervical cancer screening (AU $\$ 30,782$ adjusted cost per life year) and breast cancer screening (AU $\$ 6,600-11,000) .^{22}$

\section{How should we screen for haemochromatosis?}

The starting point for identifying individuals who would participate in a screening programme could be based on phenotype or genotype. Phenotype is assessed by investigating liver function or haematological iron values. Genotype is determined by identifying a gene mutation in HFE, either by cascade screening of relatives of index cases, or in the community without preselection.

In the past, phenotypic screening of first degree relatives has occurred on an ad hoc basis following the identification of a symptomatic proband. Suspicion of disease is based on abnormal iron studies (ie elevated serum ferritin and transferrin saturation) and confirmed by an elevated hepatic iron index on liver biopsy. Liver biopsy is also undertaken to identify the presence or absence of cirrhosis, a hallmark of irreversible and advanced disease. ${ }^{23}$ Phenotypic identification of presymptomatic homozygotes using serum iron markers is less reliable, particularly in women, and heterozygotes can only be identified using genetic analysis rather than phenotype.

Current genotype assessment in Australia is based on cascade screening of first degree relatives of affected homozygote probands. Although this is an effective way to identify further cases of haemochromatosis, siblings of affected patients may already have severe iron loading and early or even advanced symptoms of disease. In addition, ethical issues arise around the possibility of coercive interaction within a family when genetic testing in an affected relative is necessary before screening can be provided to other family members.

There are two important reasons why we believe population-based genotype screening for $\mathrm{HH}$ should not be delayed. Firstly, the prospective diagnosis of many homozygous $\mathrm{HH}$ individuals will be missed if widespread screening is not implemented before complete information is known about penetrance. Secondly, incomplete data regarding disease penetrance and prevalence is not an a priori reason not to screen since people are likely to cope well with a degree of uncertainty when counselled about risk of disease expression if the disease is preventable and/or treatable.

The ability to identify homozygous $\mathrm{HH}$ before disease onset is the greatest advantage of DNAbased testing. With the identification of the mutations responsible for haemochromatosis, DNA testing has become the method of choice to identify those with $\mathrm{HH}$. DNA samples from individuals can be obtained easily, cheaply and reliably either from blood or mouthwash samples. ${ }^{24}$

\section{Who is affected by a decision to screen the general population?}

The arguments for and against genetic screening can be viewed from the individual perspective, or from the point of view of public health policy. Both need to be considered.

\section{A) THE PUBLIC PERSPECTIVE}

From the public perspective, screening to prevent a common disease is usually a cost-effective use of the health dollar. The cost of identifying more homozygotes through DNA screening than by current phenotypic measures would be offset by the fact that it should be cheaper to prevent disease through a health maintenance programme than to treat and manage $\mathrm{HH}$ patients as they present to a specialist unit. If all persons were screened and agreed to enrol in a prevention pro- 
gramme, it is probable that symptomatic haemochromatosis would become a very rare disease. In addition, blood donated regularly to transfusion services by homozygous but healthy presymptomatic individuals as part of a health maintenance programme would be a valuable public resource.

Another important public issue is the possibility that a group is created within the population that could be discriminated against because of their genotype, even in the absence of clinical symptoms. There are data indicating that women with familial breast cancer are reluctant to accept genetic screening because of fears of discrimination in insurance. ${ }^{25}$ In the case of haemochromatosis, we suggest that discrimination would be irrational and based on a misunderstanding of disease progression, as the individual would be healthier and have a longer lifespan if tested, provided a prevention programme was accepted. Many countries are implementing legislation or agreements which prevent discrimination in insurance or employment due to genetic testing. ${ }^{26-27}$

It must be emphasised that a major educational programme of both the lay and medical communities would need to be implemented to raise awareness of issues surrounding diagnosis and management of haemochromatosis. Educational programmes would assist implementation of a successful screening programme as well as help minimise any misinformation and resultant inappropriate discrimination. Such programmes would not be simple or cheap, but any advances in understanding of genetics in one area, such as haemochromatosis, would also provide benefits in other areas of gene testing.

\section{B) THE HOMOZYGOTE'S PERSPECTIVE}

The homozygous individual clearly benefits from the implementation of screening, as development of disease can be prevented. Since compound heterozygote individuals also appear to be susceptible to disease expression, we believe these individuals also stand to benefit from having their $\mathrm{HH}$ genetic status identified.

It is possible that a small proportion of individuals with symptomatic haemochromatosis may have alternate genetic mutations to those already identified and thus would be missed by screening programmes that identify only the two common mutations. This proportion is likely to be very small in Anglo-Celtic populations, but may be greater in Southern Europeans. ${ }^{28}$ Identification of any further disease-causing mutations will increase our ability to screen for presymptomatic homozygotes.

A further issue is that unless catch-up screening is administered to the whole population, a cohort of people will be unaware of their $\mathrm{HH}$ status and may remain unidentified until presentation wi advanced disease. The increased level of educe tion in the community following the implement唐tion of a population-based screening programme would, however, serve to benefit these individuads by increasing the vigilance for $\mathrm{HH}$ disease iden fication.

\section{C) THE HETEROZYGOTE'S PERSPECTIVE}

From the perspective of the heterozygote, information regarding predisposition to iron loading $\overrightarrow{\&}$ other diseases is central to whether identificati of the heterozygote status is beneficial to the ind vidual, though it must be remembered that heterozygosity may equally be associated with. selective advantage. For instance, it has been suig gested that heterozygote women may have reproductive advantage, as they are less likely go $^{\circ}$ have iron deficiency. ${ }^{19}$

Data on the incidence of iron loading in heter zygotes is currently inconclusive. The likeliho a्d of a heterozygote developing haemochromato इ्मs in the absence of a second aggravating disea appears to be very low. Bulaj et al studied over ou thousand heterozygotes. Although $4 \%$ of femalecs and $8 \%$ of males had abnormal haematologic్. iron profiles, only one patient had evidence of mild liver damage on biopsy in the absence of a py further identifiable precipitating factor. ${ }^{19}$ This would suggest that heterozygotes are unlikely fo develop primary iron loading, but that they may have an increased risk of developing iron loading in the presence of a second disease. It has bern suggested that phlebotomy may prevent iră accumulation in heterozygote patients with a precipitating environmental factor. ${ }^{20}$ Until there ${ }_{\text {. }} \mathrm{S}$ definitive research about the propensity far disease expression in heterozygotes and whether phlebotomy in such situations is beneficial to the individual then it is difficult to inform heterozgotes appropriately. As new research is carried otzt this situation may change.

The question of whether to inform heterozizbgotes of their genetic status remains a diffichot ethical dilemma that warrants further study and consideration. Such consideration applies to many commonly inherited diseases, since we all carryaㅡ number of recessive genes that are not clinically important unless two persons with the sanife recessive mutation have children.

With regard to reproductive issues, sing haemochromatosis is a preventable disease, it $\mathrm{gs}$ unlikely that a heterozygote couple at risk of hy ing a child who is homozygous for the mutation would choose prenatal diagnosis and terminate affected pregnancy. It is unlikely that either the 
medical or lay community would support termination of an affected fetus since the disease is adult-onset and can be compatible with normal health if preventive strategies are appropriately implemented.

We believe the current cost of informing heterozygotes in both financial and psychological terms is too high. Not only would the number of people needing counselling increase greatly but the complexity of counselling would increase dramatically because not enough is known about the risk of disease expression. In the future the community may desire such information and be better equipped to deal with such complex genetic data, but the initial introduction of populationbased heterozygote screening alongside homozygous screening would only serve to confuse such a programme.

Offering information on carrier status would massively increase the cost of education and counselling, and risk creating a group of "worried well". When more is known about the medical implications of heterozygosity, decisions about whether to inform individuals of their status should become clearer. Cascade screening of relatives of probands currently identifies heterozygotes, but whether individuals are informed of their carrier status appears to be individualised by the caring physician. At this point we would not advocate informing heterozygotes identified by population screening of their status unless requested by the individual or dictated by the clinical setting.

\section{Who and when should we screen?}

It is not clear whether it is discriminatory to offer screening only to those who are in a high-risk ethnic group, omitting to screen those in other groups. Even if it appears costly to offer screening to those in low-risk populations (which, in the Australian context, would include those of Aboriginal, South East Asian and African origin) this may have significant implications in a city with much ethnic mixing, such as Melbourne. Although the cost of needless screening is an issue, so too is perceived and real discrimination against those who are not screened, with all the problems in defining ethnicity in a multicultural environment.

Implementation of population-based genetic screening will be most beneficial if administered to a receptive population and at a time when the cost-benefit ratio is most favourable. Screening to detect presymptomatic homozygotes can be directed at three main groups: neonates, adolescents and young adults.

Neonates are an easy group to target because efficient and acceptable screening is already in place for phenylketonuria and other serious diseases affecting neonates. It would be logistically simple to add a further test to those currently performed. Screening in the neonatal period ensures that homozygotes identified will definitely be presymptomatic. However, neonatal screening means there is a long lead time before the information is relevant and preventive measures can be implemented, as regular phlebotomy would be unlikely to be organised before the infant was at least 20 years of age. Parents of presymptomatic homozygous individuals may perceive their child as unwell. Parents may also implement inappropriate care such as eliminating iron from a growing child's diet. Many years of individual follow-up will be required to ensure that appropriate preventive strategies are implemented at the appropriate age.

If we compare haemochromatosis with cystic fibrosis (CF), a disease for which neonatal screening for all is already in place in Australia, we can say:

- $\mathrm{HH}$ is more common than CF ( 1 in $300 \mathrm{v} 1$ in 2,500);

- $\mathrm{HH}$ is a preventable disease while $\mathrm{CF}$ is not;

- prenatal testing for $\mathrm{HH}$ is not likely to be implemented because (unlike CF) it is both preventable and treatable;

- a single mutation accounts for more than $80-90 \%$ unlike CF where there are many mutations, the most common of which accounts for about $70 \%$ of mutations.

All parents of homozygote $\mathrm{HH}$ infants would be obligate heterozygotes and a small percentage would be homozygous for $\mathrm{HH}$ by chance. An added benefit of neonatal screening would be the identification through reverse cascade screening of further presymptomatic homozygous individuals since parents, as well as aunts and uncles, of newborns are likely to be young adults. Neonatal testing may be an option in the future when more is known about disease prevalence and penetrance and if and when there is increased awareness of $\mathrm{HH}$ in the community.

The advantage of screening adolescents is their potential to take responsibility for their own health and any preventive strategies that need implementation. Unfortunately adolescents can be susceptible to stigmatisation through peer pressure. Knowledge of $\mathrm{HH}$ homozygosity may initiate inappropriate reactionary high-risk behaviour in the individual. In addition, this group is difficult to access since school-based screening may not be acceptable to parents or school authorities.

Screening of young adults (20-30 year olds) has many ethical advantages. Young adults are autonomous and capable of giving consent, and if they have counselling will be aware of the useful- 
ness of the test and the possible implications for relatives and with regard to insurance. They also are likely to be motivated to enter into a prevention programme. However, a small minority of cases may already have liver damage below the age of 30 .

We favour screening of young adults with the proviso that health maintenance programmes be implemented for individuals identified as homozygous for $\mathrm{HH}$. Ongoing support, education and counselling would help ensure that homozygous individuals remain informed of the latest research developments and have ready access to information regarding health maintenance. However, public education programmes and resolution of issues of stigmatisation need to be addressed before implementation of population-based screening. Participation in a screening programme would need to be completely voluntary.

\section{Conclusions}

Haemochromatosis is an ideal disease in which to implement population-based genetic screening because it is both common and preventable. On balance, the public and the individual stand to gain from the implementation of genetic screening. At this point in time, screening of young adults would be the most readily accepted by the community and the most efficient to implement. Education of the medical and lay communities would be vital to the success of such a programme and pilot studies of population-based screening would help address many of these issues. Before a widely applied programme could be implemented, issues regarding funding and implementation of health maintenance programmes for all homozygous individuals identified would need to be fully explored. In addition, issues around the health status of heterozygotes need further consideration. Finally legislation against geneticbased discrimination and misuse of genetic information should be given priority before embarking on a programme of population-based screening.

Katrina Allen MBBS, BMedSc, FRACP is a Paediatric Gastroenterologist at The Murdoch Institute, Royal Children's Hospital, Parkville, Victoria, Australia. Robert Williamson PhD, FRCPath, FRCP (Edin), is Director of The Murdoch Institute.

\section{References}

1 Modell B, Moddell M. Towards a healthy baby: congenital disorders and the new genetics in primary health care. New York: Oxford University Press, 1992.
2 WHO scientific group report. Control of hereditary diseasps. Geneva: WHO, 1996.

3 Burke W, Thomson E, Khoury $\mathbf{M}$ et al. Genetic testing $\overrightarrow{\text { foj }}$ hereditary hemochromatosis and its implications. Fournal of . The American Medical Association 1998;280,2:172-7.

4 Williamson R. Universal community carrier screening $\overrightarrow{\overline{\text { ⿷⿱ }}}$ cystic fibrosis. Nature Genetics 1993;3:195-201.

5 DudokdeWit AC, Tibben A, Duivenvoorden HJ et al. Distress in individuals facing predictive DNA testing for autoso dominant late-onset disorders: comparing questionnaire res $\overrightarrow{\underline{T}}$ with in-depth interviews. American fournal of Medical Gen 1998; 75,1:62-74.

6 Morris MJ, Tyler A, Lazarou L et al. Problems in genetic peediction for Huntington's disease. Lancet 1982;2(8663):601-9?.

7 Adams PC, Kersatz AE, Valberg LS. Clinical presentationdf hemochromatosis. American fournal of Medicine 1991;90:445-9.

8 Powell LW. Hemochromatosis, the impact of early diagnegis and therapy. Gastroenterology 1996;110:1304-7.

9 Niederau C, Fischer R, Purschell A et al. Long-term survivatin patients with hereditary haemochromatosis. Gastroenterola 1996;110:1107-1119.

10 Adams PC, Agnew S. Alcoholism in hereditary hemochrorfittosis revisited: prevalence and clinical consequences amongst homozygous siblings. Hepatology 1996;23:724-7.

11 Feder JN, Gnirke A, Thomas W et al. A novel MHC class I-A A gene is mutated in patients with hereditary hemochromatos?s. Nature Genetics 1996;13:399-408.

12 Jouanolle AM, Fergelot P, Gandon G et al. A candidate gene for hemochromatosis: frequency of the $\mathrm{C} 282 \mathrm{Y}$ and $\mathrm{H} 63 \mathrm{D}$ mutations. Human Genetics 1997;100:544-7.

13 Jazwinska EC, Cullen LM, Busfield F et al. Haemochromatơsis and HFE. Nature Genetics 1996;13:399-408.

14 The UK Haemochromatosis Consortium. A simple genetic $\overrightarrow{\text { test }}$ identifies $90 \%$ of UK patients with haemochromatosis. 1997;41,6:841-4.

15 Legget BA, Halliday JW, Brown NN et al. Prevalence $\square_{f}$ haemochromatosis amongst asymptomatic Australians. Brish fournal of Haematology 1990;74:525-30.

16 Powell LM, Bassett ML. Haemochromaotisis: diagnosis $\stackrel{\supset}{\rightleftharpoons}$ management after the cloning of the HFE gene. Australian fitd New Zealand fournal of Medicine 1998;28:159-63.

17 Burke W, Press N, McDonell SM. Hemochromatosis: genefss helps to define a multifactorial disease. Clinical Genes 1998;54:1-9.

18 Crawford HG, Jazwinska EC, Cullen LM et al. Expression of HLA-linked hemochromatosis in subjects homozygous or therozygous for the C282Y mutation. Gastroenterology 1998;184: 1003-8.

19 Bulaj ZJ, Griffen LM, Jorde LB et al. Clinical and biochemigal abnormalities in people heterozygous for hemochromatosis. New England fournal of Medicine 1996;335:1799-1805.

20 George DK, Goldwurm S, MacDonald GA et al. Increastd hepatic iron concentration in nonalcoholic steatohepatitis is associated with increased fibrosis. Gastroenterology 1998;1ळ4: 311-18.

21 Bassett ML, Leggett BA, Halliday JW et al. Analysis of the c8st of population screening for haemochromatosis using biochemical and genetic markers. fournal of Hepatology 1997;27: 517-84.

22 Carter R, Glasziou P, van Oortmarssen G et al. Codseffectiveness of mammographic screening in Australia. Aust ian Fournal of Public Health 1993;17,1:42-50.

23 Bacon BR. Diagnosis and management of haemochromatosis. Gastroenterology 1997;113: 995-9.

24 Lench N, Stanier P, Williamson R. Simple non-invasive methef to obtain DNA for gene analysis. Lancet 1988;1:1356-8.

25 Bekendorf JL, Reutenauer JE, Hughes CA et al. Patiests' attitudes about autonomy and confidentiality in genetic testirg for breast-ovarian cancer susceptibility. American fournd Medical Genetics 1997:73:296-303.

26 Otlowski M, Chalmers D, Skene L et al. Discussion paperr. Implications of the human genome project for Australian insurance law and practice. Canberra: Australian Research Council, 19 $\overline{g g}$.

27 Hudson KL, Rothenberg KH, Andrews LB et al. Gen@ic discrimination and health insurance: an urgent need \$or reform. Science 1995;270:391-3.

28 Piperno A, Sampietro M, Pietrangelo A et al. Heterogeneity hemochromatosis in Italy. Gastroenterology 1998;114:996-1002. 